\title{
CDK8 inhibitor: Senex's best thing
}

\section{By Michael J. Haas, Senior Writer}

Chemotherapy is used to treat virtually every tumor type, but the drugs can blunt their own efficacy by inducing paracrine activity. Now, an international team led by Senex Biotechnology Inc. has mouse data showing that a cyclin dependent kinase 8 inhibitor can block the negative effects of at least one chemotherapeutic-doxorubicin. ${ }^{1}$

The unanswered questions include whether the cyclin dependent kinase 8 (CDK8) inhibitor enhances the efficacy of additional chemotherapies and targets cancer stem cells.

Doxorubicin is one of several chemotherapies that exert their efficacy by directly damaging DNA. However, this damage can induce the secretion of factors by host and tumor cells-so-called paracrine activitiesthat promote cancer cell growth, metastasis and drug resistance..$^{2-5}$ In addition, studies have shown that paclitaxel, which acts by stabilizing microtubules instead of damaging DNA, can also promote tumor invasion and metastasis. ${ }^{6-8}$

\section{"The potential for} chemotherapeutic drugs to simultaneously inhibit tumor growth and stimulate or activate host mechanisms that may undercut the drugs' efficacy and hasten relapse is a topic of renewed-and growing - interest." - John Ebos, Roswell Park Cancer Institute
Although the molecular mechanisms that drive paracrine activities have been poorly understood, one clue emerged about a decade ago when researchers led by Igor Roninson, who was then at the University of Illinois at Chicago, found that cyclin-dependent kinase inhibitor $1 \mathrm{~A}$ (p21, Cip1; CDKN1A; CIP1) regulated cellular responses to DNA damage.

The Chicago group showed that in a human cancer cell line, doxorubicin induced $\mathrm{p} 21$, which in turn upregulated multiple tumorpromoting antiapoptotic, mitogenic and angiogenic proteins. ${ }^{9,10}$ Although p21 is known to regulate many transcription factors and several cyclin dependent kinases (CDKs) involved in transcription and the cell cycle, the studies did not elucidate p21's precise mechanism of action in promoting paracrine activities.

In the new study, a Roninson-led team from Senex set out to investigate the tumor-promoting pathways downstream of p21 to identify potential cancer targets.

First, the group used a p21-overexpressing cancer cell line developed by Roninson's previous team to screen compound libraries for small molecules that inhibited p21-induced expression of tumor-promoting factors. Several hits contained a 4 -aminoquinazoline core, and optimization of this structure resulted in the lead compound, Senexin A.
Because Senexin A resembled known protein kinase inhibitors, the team screened the compound against 442 kinases and found it was a selective nanomolar inhibitor of CDK8 and a closely related isoform, CDK19 (CDC2L6). Additional in vitro experiments confirmed that the two kinases act downstream of p21 to induce the expression of multiple tumor-promoting proteins.

Next, the team injected mice with a human lung cancer cell line and mouse embryonic fibroblasts, which have paracrine activities similar to those of tumor stromal cells in patients with cancer. In these xenograft models, Senexin A plus doxorubicin decreased tumor growth compared with doxorubicin alone.

Lastly, the group used a computational tool to search online microarray data for links between expression of the two kinases and survival in breast and ovarian cancer patients. They found that levels of each kinase correlated with poor relapse-free survival in both types of cancer, including ovarian cancers treated with DNA-damaging platinum chemotherapies. These findings suggest CDK8 and CDK19 are associated with the failure of such chemotherapies in the clinic and bolster the rationale for these kinases as cancer targets, the team wrote in its report in the Proceedings of the National Academy of Sciences.

The study also shows that Senexin A can block the oncogenic effects of CDK8 that do not require $\mathrm{p} 21$, such as the wingless-type MMTV integration site (WNT) and $\beta$-catenin (CTNNB1) pathway, Roninson told SciBX. "Thus, our compound inhibits CDK8 and its effects regardless of p21 expression in cells," he said. CDK8 is upregulated in some colon and gastric cancers in which it activates WNT and

CTNNB1 signaling. ${ }^{11,12}$

Roninson is founder, president and CSO of Senex and chair of translational cancer therapeutics and professor of pharmaceutical and biomedical sciences at the University of South Carolina's South Carolina College of Pharmacy. He formerly was professor of molecular genetics and head of the Division of Molecular Oncology at the University of Illinois at Chicago.

The team also included researchers at the Ordway Research Institute, Rensselaer Polytechnic Institute, The Scripps Research Institute, the Hungarian Academy of Sciences and the University of Athens Medical School.

"The potential for chemotherapeutic drugs to simultaneously inhibit tumor growth and stimulate or activate host mechanisms that may undercut the drugs' efficacy and hasten relapse is a topic of renewed-and growing-interest," said John Ebos, assistant professor of oncology at Roswell Park Cancer Institute. "The potential for CDK8 blockade with Senexin A raises an interesting therapeutic possibility" for countering the cancer-promoting effects of chemotherapy.

William Nelson, director and professor of oncology at The Johns Hopkins University and director of JHU's Sidney Kimmel 
Comprehensive Cancer Center, agreed. He said the complexity of intercellular signaling in the tumor microenvironment has made it challenging to identify the key pathways that are involved in the tumorpromoting effects of chemotherapies. "The nomination of CDK8 and CDK19 as therapeutic targets for undermining survival signaling in cancers is intriguing," he told SciBX.

Yuval Shaked, assistant professor and senior lecturer of molecular pharmacology at the Technion-Israel Institute of Technology's Ruth and Bruce Rappaport Faculty of Medicine, added that "an agent like Senexin A with such a specific target is potentially promising for clinical investigation since the probability of severe toxic side effects is low."

\section{Figure eights}

Other cancer researchers said the safety and specificity of Senexin A was not yet clear.

Nelson cautioned that inhibiting CDK8-

\section{"We anticipate that our CDK8 inhibitors may be effective as single agents by blocking the tumor-initiating capacity of cancer stem cells." \\ - Igor Roninson, \\ Senex Biotechnology Inc.}

\section{Larger circles}

In addition to the monotherapy studies, Ebos and Shaked want to see Senexin A tested in combination with doxorubicin in additional animal models of cancer, including metastatic cancers.

Shaked added that "it would be interesting to evaluate Senexin A in combination with other DNA-damaging drugs, such as cisplatin," or chemotherapies that do not directly damage DNA, such as paclitaxel.

He said studies in the past three years have shown that $\mathrm{p} 21$ is upregulated in cancer stem cells. ${ }^{13,14}$ Those results suggest compounds like Senexin A that target pathways downstream of p21 might have the added benefit of making cancer stem cells more susceptible to chemotherapy and thereby delaying or preventing tumor regrowth and relapse, he said.

Roninson agreed, citing a February study by a team from Roche's Genentech Inc. unit that suggested CDK8 plays a role in maintaining the cancer stem cell phenotype. ${ }^{15}$ "We anticipate that our CDK8 inhibitors may be effective as single agents by blocking the tumor-initiating capacity of cancer stem cells," he said.

Roninson said Senex is testing Senexin B-an optimized version of Senexin A-in animal models of lung, breast, colon and prostate cancer in combination with doxorubicin and other undisclosed chemotherapies. The company also is testing Senexin B as a single agent to treat cancer, based in part on the ability of CDK8 inhibitors to block the WNT and CTNNB1 signaling pathway.

Additionally, Senex is conducting IND-enabling studies of Senexin $\mathrm{B}$ and seeking a corporate partner for clinical development of the compound, Roninson said.

Senex has patented the findings reported in PNAS and subsequent optimized inhibitors.

Haas, M.J. SciBX 5(33); doi:10.1038/scibx.2012.858 Published online Aug. 23, 2012

\section{REFERENCES}

1. Porter, D.C. et al. Proc. Natl. Acad. Sci. USA; published online Aug. 6, 2012; doi:10.1073/pnas.1206906109

Contact: Igor B. Roninson, Senex Biotechnology Inc., Columbia, S.C. e-mail: roninsoni@sccp.sc.edu

2. Liu, D. \& Hornsby, P.J. Cancer Res. 67, 3117-3126 (2007)

3. Coppé, J.P. et al. PLoS Biol. 6, e301; published online Dec. 2, 2008; doi:10.1371/journal.pbio.0060301

4. Daenen, L.G.M. et al. Cancer Res. 71, 6976-6985 (2011)

5. Haas, M.J. SciBX 4(44); doi:10.1038/scibx.2011.1226

6. Quintavalle, M. et al. Sci. Signal. 4, ra49 (2011)

7. Gingis-Velitski, S. et al. Cancer Res. 71, 6986-6996 (2011)

8. Haas, M.J. SciBX 4(31); doi:10.1038/scibx.2011.870

9. Chang, B.-D. et al. Proc. Natl. Acad. Sci. USA 97, 4291-4296 (2000)

10. Chang, B.-D. et al. Proc. Natl. Acad. Sci. USA 99, 389-394 (2002)

11. Firestein, R. et al. Nature 455, 547-551 (2008)

12. Kim, M.-Y. et al. Int. J. Oncol. 38,1375-1383 (2011)

13. Viale, A. et al. Nature 457, 51-56 (2009)

14. Benayoun, L. et al. Stem Cells; published online Aug. 20, 2012; doi:10.1002/stem.1170

15. Adler, A.S. et al. Cancer Res. 72, 2129-2139 (2012) 


\section{ANALYSIS}

COMPANIES AND INSTITUTIONS MENTIONED

Genentech Inc., South San Francisco, Calif.

Hungarian Academy of Sciences, Budapest, Hungary

The Johns Hopkins University, Baltimore, Md.

Ordway Research Institute, Albany, N.Y.

Rensselaer Polytechnic Institute, Troy, N.Y.

Roche (SIX:ROG; OTCQX:RHHBY), Basel, Switzerland

Roswell Park Cancer Institute, Buffalo, N.Y.

\section{TARGETS \& MECHANISMS}

Sanofi (Euronext:SAN; NYSE:SNY), Paris, France The Scripps Research Institute, La Jolla, Calif. Senex Biotechnology Inc., Columbia, S.C.

Technion-Israel Institute of Technology, Haifa, Israel University of Athens Medical School, Athens, Greece University of Illinois at Chicago, Chicago, III. University of South Carolina, Columbia, S.C. 